\title{
Discussion of methods in building and validating a model: example of amino acid metabolism in ruminants.
}

\author{
P Lescoat 1,2, A Danfær 1, D Sauvant 2 \\ 'Danish Institute of Animal Science, Research Center Foulum, P.O. Box 39 DK-8830 TJELE, Denmark \\ 2 Institut National Agronomique Paris-Grignon, Department of Animal Science, 16, rue Claude Bernard, \\ 75231 Paris Cedex 05, France
}

\begin{abstract}
Summary - A methodological approach of mechanistic modelling is proposed. Firstly, the goals and features of the model are defined. The qualitative flux diagram is described. Its translation into mathematical equations is presented. Some options to determine the model parameters are proposed. The mathematical integration and some limits are studied. Theoretical validations are made with an emphasis on sensitivity analyses. Several possibilities for external validation are shown. These different steps are illustrated through the development of a mechanistic model of the amino acid metabolism in the lactating cow. The decisions taken at each step of the modelling process are discussed and their limits are settled. The conclusions underlined the difficulty to endup with a final model and proposed an interdisciplinary approach as a possible solution. However, the models are very important through their construction since they underline the areas where there is a gap of knowledge and therefore they are an interesting tool to orientate future experiments. Moreover, these models can also be used pratically if they have been validated.
\end{abstract}

\section{Introduction}

Current systems of cattle nutrition mostly deal with the digestion of feedstuffs (Tamminga et al, 1994). These systems predict the available quantities before or after absorption in the digestive tract of nutrients either aggregated or individually (lysine and methionine in the French PDI system (Rulquin et al, 1993) or ten essential amino acids in the Cornell system (O'Connor et al, 1993)). Intermediary metabolism is generally treated with a factorial approach to balance supply with requirement (INRA, 1988; Tamminga et al, 1994). Even though a nutrient-response system to amino acid availability has been built by Rulquin and co-workers (Rulquin et al, 1993), none of these models describe or simulate the fate of amino acids. Events between the intestinal wall and the mammary glands are summarized in a "black box" with a constant efficiency. Consequently, events such as protein mobilization or amino acid involvement in gluconeogenesis are not taken into account. An approach to cope with these phenomena is to build a more mechanistic model of the amino acid fluxes in the intermediary metabolism of the lactating cow. In this paper, some features of a model dedicated to the simulation of the amino acid fate in the intermediary metabolism of the lactating cow are presented. However, it has to be kept in mind that this model is in its early stage of development. Therefore, this paper is only a discussion of some methodologies used to build and to validate a mechanistic model and of some problems encountered during this model development.

\section{Definition of the model}

\section{Aim of the model}

This model is built to deal with the protein and amino acid fluxes in the lactating ruminant during a lactation period. This model should be able to simulate the fate of amino acids from the intestinal wall to the mammary glands. This goal is more restricted than the whole-animal models proposed by Baldwin et al (1987a) and Danfær (1990). These two works simultaneously studied the energy and protein metabolism with the same degree of mechanisticity. Our approach focused deeper on amino acid metabolism. Consequently, this model could not simulate all variations induced by lipid metabolism.

\section{A compartmental model}

The frame of the model is based on compartments of tissues and metabolites and of 
their relationships. This approach is suitable to our aim since fluxes of matter and informations have to be predicted by this model.

\section{A mechanistic model}

This model is mechanistic according to the comparison between its aim and structure (Forbes and France, 1993). To predict a phenomenon at a given level, here the whole animal amino acid fate, protein and amino acid utilizations are described at organ or even cellular levels. However, the concept of mechanistic has a relative meaning. All parameters in the model are estimated from literature values and they can be considered as the aggregation of sub-phenomena, which is in fact the case in all mechanistic model.

\section{A dynamic model}

Since the driving force of this model is milk production level and that this value is determined by the number of days in lactation, the model is dynamic (Forbes and France, 1993).

\section{A deterministic model}

All the parameters in the dynamic differential equations describing the behaviour of the model have constant values. Consequently, the model is deterministic. This is raising the question of the knowledge concerning each parameter (see section "Parameterization»).

\section{Building of the model diagram}

The mainframe of the model is based on a work between Sauvant and Phocas (Sauvant et al, unpublished). This first model intends to describe simply the energy metabolism of the lactating cow. It is organized with two different levels: an operating subsystem and a regulating one (Sauvant, 1994). The operating subsystem includes all the compartments, their

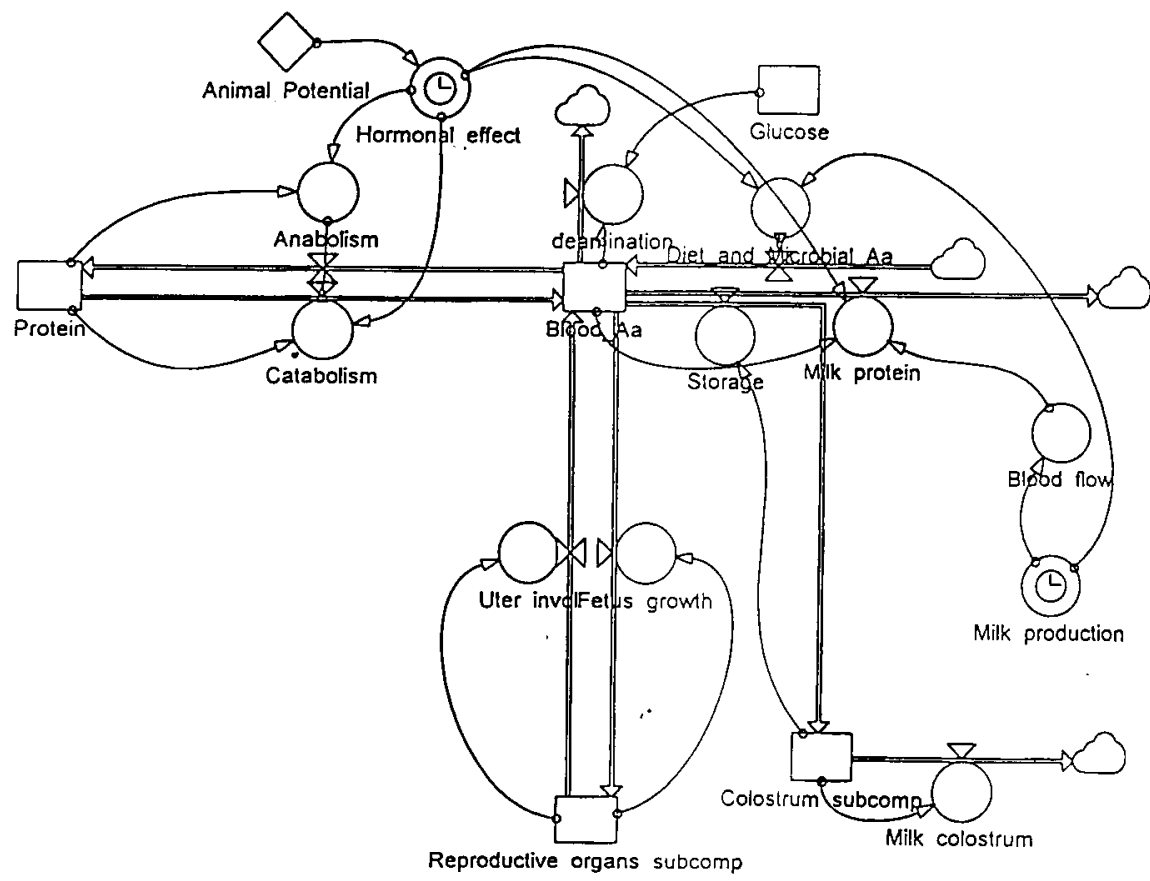

Figure 1. Forrester diagram of the lactating cow model: protein section. 
exchanges through the nutrient fluxes, the inputs from the digestive tract and the outputs as milk protein and fat. The regulating subsystem influences the fluxes and compartments by simulating only homeorhetic regulations. A Forrester diagram of the model is presented Figure 1.

\section{The operating subsystem}

Since this presentation aimed to be methodological, the following sections of this paper deal only with protein and amino acid pools.

\section{Choice of the compartments}

The number of compartments describing amino acid and protein metabolism can vary broadly. However, the proposal allowing the lowest number of parameters to estimate amino acid and protein metabolism is preferred. Nevertheless, the model should mimic correctly actual protein metabolism, therefore a major problem is to reach the right compromise.

The amino acid part of the model consists of three compartments: the protein compartment, the blood amino acid compartment and the reproductive organs (Figure 1).

The largest one is the protein compartment. It includes every protein in the body except those from the reproductive organs and the blood. This can be considered as highly aggregated. It is documented that this compartment is not homogeneous on a dynamic standpoint. Therefore, it should not be considered as a single compartment. It could have been divided into two: muscle tissues and portal drained viscera, as in Baldwin et al (1987a). This two-compartments approach seems relevant for the lactating cow since these different protein masses are largely independently regulated during lactation: at the beginning of lactation, portal drained viscera mass increases largely whereas the muscle is submitted to a high rate of catabolism. Another difference is the protein turnover rate which is several times higher in the portal drained viscera than in the muscle tissues. A less aggregated approach could have been done, since each tissue or organ containing protein could be considered individually regarding their different fractional synthesis rate (Lescoat et al, 1995). Nevertheless, the possibility of one compartment by tissue and organ is not easily applicable due to the lack of quantitative knowledge. In the early stage of model development and according to the data available, the one protein compartment option was chosen bearing in mind its limits.

A second protein compartment is the reproductive organs. It delivers protein at the beginning of lactation during uterine involution and it takes up amino acids in the last gestation months when foetus protein requirements become significant.

The third compartment is blood amino acids. Theoretically, this compartment includes all amino acids or small peptides (up to $1500 \mathrm{D}$ ) in the blood (Backwell, 1994). Since the importance of each component of this compartment has only begun to be investigated (MacRae et al, 1995), they are all put in the same pool. Their behaviours as carriers of amino acids between the different tissues and organs have to be studied. Intracellular amino acid could have been added in the mammary glands. It describes a latence pool before milk protein synthesis (France et al, 1995) for leucine. However, a minimal configuration was chosen for this early version.

This model does not treat amino acids individually and has severe limits. Nevertheless, this simple structure was chosen since more precise knowledge was missing to parameterize the amino acid compartments.

\section{Fluxes between the compartments}

Compartments are receiving inputs from the outside of the compartment and they are delivering outputs. Each of these fluxes represents part of the metabolism.

There are only two fluxes in the protein compartment. The input is the anabolic flux, it covers two different phenomena: protein turnover rate and protein accretion. The first one requires amino acids to replace enzymes and structural proteins although there is an intracellular recycling of degraded proteins (Smith and Sun, 1995). The protein accretion occurs in different situations: in first lactation, since the animal is still growing, and in late lactation, because there is a reconstitution of the body reserves (Belyea et al, 1978). The output from the protein compartment is the catabolic flux. It includes a component due to the protein turnover rate, nearly the same as in the anabolic flux. Nevertheless, the major proportion of this flux could be linked with amino acid mobilization at the beginning of the lactation. The input comes from and the output comes to the blood amino acid compartment. 
An important issue in this model is to determine the size of these different fluxes. If the turnover is taken totally into account (i.e. the molecules that are recycled are released in the amino acid blood compartment and immediately taken-up again), then the total fluxes are highly increased. Nevertheless, this recycling could be assumed to be intracellular and consequently not taken into account in the fluxes to the blood.

The reproductive organ compartment has two fluxes: one output and one input. The output mimics the uterus involution at the beginning of lactation. After a couple of weeks, this compartment is empty. Symmetrically, during the gestation period, the requirements for the foetus and its annexes are increasing and they are pumping amino acids into the blood compartment (Battaglia, 1992). Consequently, this compartment becomes competitive with the milk protein synthesis process and the reconstitution of body storages for the next lactation-gestation cycle. This compartment only exists during a small period compared with the simulation length.

The blood amino acid compartment is the most open one. It receives three inputs and delivers five outputs. The inputs are the amino acids from the intestinal lumen, the catabolized proteins from the protein compartment and the flux from the reproductive organs. The outputs are the amino acids taken-up by the mammary glands for milk protein synthesis, the amino acids deaminated for gluconeogenesis or ketogenesis, the amino acids for the protein compartment anabolism and the amino acid taken by the reproductive organs. The different fluxes are not easily calibrated. As an example, the extent of the withdrawal of the amino acid by the intestinal wall between the intestinal lumen and the portal vein is not settled. Tagari and Bergman (1978) found high levels of amino acid metabolization in the intestinal wall whereas MacRae et al (1995) showed that the portal drained visceral tissue mainly uses arterial amino acids. Consequently, during the validation of this model, the effect of the variation of this metabolization will have to be tested.

This minimal model will only include fluxes that can be measured or estimated.

\section{Regulations of the fluxes}

The fluxes represent the compartment exchanges and interactions between them. Each flux is regulated even passively by the

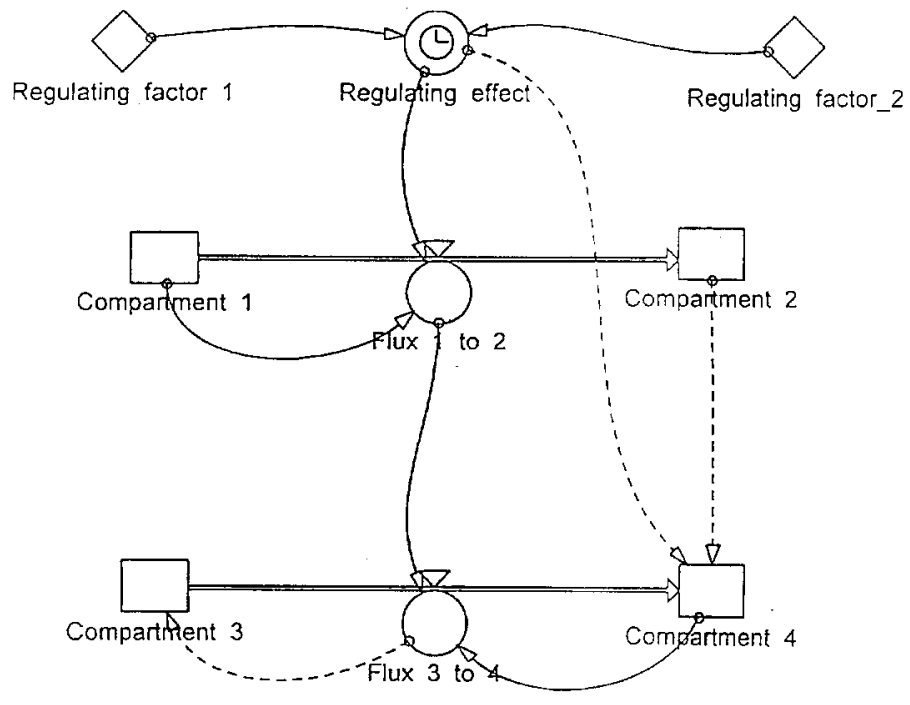

Figure 2. Relationships between compartments. 
mass action law. The regulations are critical points in the definition of a model since they are the means to transfer the driving forces.

Several kinds of influences can alleviate a flux between two compartments (Figure 2). Every combination between an hormonal action, a flux and a compartment size can influence another flux. All those regulations in the operating system could be linked by analogism with the metabolic control theory developed to study biochemical pathway control (Kacser and Burns, 1979). The main problem raised by these authors is that the influences of substrates, enzymes, other metabolites on one flux are varying in a complex shape: they stated that «Fluxes and metabolite levels (compartment size in our case) are systemic properties". Therefore, even though the qualitative effects are known, their relationships in vivo are hardly ever quantified. For example, a major research area in intermediary metabolism in ruminants is the gluconeogenesis precursor partition. Propionate, amino acids, glycerol and lactate are used to various extents to produce glucose. The relative proportion of each precursor depends on its availability for propionate and on several hormonal action for the other precursors. These proportions are key-points regarding amino acid metabolism since the contribution of amino acids to gluconeogenesis varies from 2 to $50 \%$ of the requirements and averages $20 \%$ (Danfær et al, 1995). To represent this uncertainty, two possibilities are available. The first one is to take into account the only variables in the model quantitatively known as influencing amino acid flux to gluconeogenesis, glucose and propionate. This solution ignores all the interactions between nutrients and hormones and the homeorhetic regulation. The second solution is to build a theoretical model of nutrient partition driven by quantitative rules, like a target-glucose concentration with limitations for the allowed variations and by limits of each nutrient possible contribution. This second solution leads to an unknown system. In our model, even though the second solution is attractive, the minimal first one was chosen. From this example, it comes out that even if the complexity of the flux regulation is kept in mind, it could only be roughly taken into account.

The extent of the fluxes can be determined by a regulating subsystem (Figure 2 , Sauvant,
1994). Depending on the prioritary function at a given period, the partition of the nutrients between different purposes can change. Hormones are transmitting the homeorhetic driving forces to the fluxes. The interactions between hormones are not known. Therefore, it seems difficult to alleviate the fluxes directly by using the actual hormone concentrations as did Danfaer (1990). An alternative solution is to build a theoretical system with a couple of anabolic and catabolic hormonal effects varying with the maximal milk production level of the animal (Sauvant, 1994). However, the hormonal effects are difficult to quantify because the subsystem is theoretical. Moreover, these effects are hidden in the literature by other variables as levels of nutrition, animal ages, experimental designs and methods of measurements. For example, concerning protein mobilization and storage, Gibb et al (1992) observed a low protein mobilization in the lactating cow with a significant influence of the diet composition. However, they stated that relevant results on protein mobilization are difficult to obtain due to technical problems and to different dynamics of tissues and organs included in the protein compartment. These results underline the critical work in linking this regulating subsystem and the fluxes in the operating subsystem.

\section{The regulating subsystem}

A regulating subsystem is added to cope with the homeorhetic regulations (Figure 1). Therefore, this model could be classified in the fifth level of the system classification proposed by Le Moigne (1990): this system is not only based on fluxes and compartments but also there is a subsystem of decision which alleviates the fluxes between compartments. It influences the operating subsystem according to the genetic merit of the animal and the prioritary function changes in the animal metabolism during the whole lactation. A series of hypotheses have been used to build this theoretical subsystem of hormonal effects as described in the above section and they are explained in details by Sauvant (1994). As an example, this subsystem could influence the amino acid uptake by the mammary glands according to the «pull» hypothesis. This assumption states that this is the activity of the mammary gland metabolism which determines the quantity of amino acid taken up by this 
organ (Knight et al, 1994).

The regulating subsystem drives several key-fluxes in the operant subsystem. The whole complex system of endocrine and neural mechanisms are aggregated in this couple of hormonal effects. This is obviously an oversimplification but for example, an alternative approach using the ratio between a reference glucose level and the actual glucose concentration (Baldwin et al, 1987a) seems more a homeostatic than a homeorhetic regulation.

\section{Mathematical writing of the model}

\section{Ordinary Differential Equations}

The size of a compartment is identified with a state-variable. The algebraic sum of the fluxes during an interval of time determines the variation of the state-variable during the same period. A tool to translate these over-time changes in a compartmental modelling is the ordinary differential equation (Danfaer, 1990) There are three differential equations in the protein part of the lactating cow model, one for each compartment.

\section{Inclusion of the assumptions in the equations}

The functions determining the fluxes in the differential equations have to translate quantitatively the hypothesis assumed in the qualitative section. A large choice of equations are available (Table I, adapted from Freetly et al, 1993). They can be classified into two subspecies: mass-action or Michaelis-Menten laws.

The mass action flux is regulated by the substrate quantity. The multiplying factor is either constant or influenced by another flux or a hormonal effect. This is the case for the protein compartment anabolism except that this is a mass-action law of the product.

The Michaelis-Menten law is characterized by limits of the absolute flux: either as a saturation or an inhibition of the process regulating the flux. An analogy could be made with one controlling enzyme activity as the bottleneck of a whole metabolic pathway (Kacser and Burns, 1979). As for the biochemical level, substrates and products or hormones can enhance or inhibit the flux. Up to five substances were proposed by Freetly et al (1993) to influence one flux. One limit of this approach is that a major part of the parameter values for these Michaelis-Menten regulations have been determined in vitro or in vivo in other animal species.

A third parameterization to include the hypotheses in differential equations has been used for the lactating cow model. An example can be given by the amino acid uptake in the mammary glands which has been commonly measured by arteriovenous difference technique (Lescoat and Sauvant, 1994).

Table I. Examples of differential equation fluxes parameterization (adapted from Freetly et al, 1993) 1

Mass action law

$F_{A, B}=K_{A, B} S_{A}$

$F_{A, B}=K_{A, B} * S_{B}$

$F_{A, B}=K_{A, B} * S_{A} *\left(C /\left[S_{B}\right]\right)$

$F_{A, B}=K_{A, B} S_{A} *(H E) E$

Michaelis-Menten law

$F_{A, B}=V_{A, B} /\left(1+\left(K_{A} /\left[S_{A}\right]\right) E\right)$

$F_{A, B}=V_{A, B} /\left(1+\left(\left[S_{A}\right] / K_{A}\right) E\right)$

$F_{A, B}=V_{A, B} /\left(1+\left(K_{A} /\left[S_{A}\right]\right) E+\left(\left[S_{B}\right] / K_{B}\right)^{F}\right)$

$F_{A, B}=V_{A, B^{*}}(H E) E /\left(1+\left(K_{A} /\left[S_{A}\right]\right)^{F}\right)$

$\left.F_{A, B}=V_{A, B} /\left(1+\left(K_{A^{*}}(H E)^{E}\right) /\left[S_{A}\right]\right)^{F}\right)$

First order kinetic, influence of the substrate

First order kinetic, influence of the product

Feedback control, influence of the substrate and product Hormonal effect influence

${ }^{1} F_{A, B}$ : flux from $A$ to $B(m o l / d), K_{A, B}$ : Fractional flux rate $(/ d), S_{A}$ and $S_{B}$ : Sizes of the compartments $A$ and $B$ (mol), HE: hormonal effect, $C$ : constant value (mol/l), $V_{A, B}$ : Maximum flux rate (mol/d), $K_{A}$ and $K_{B}$ : Affinity constants $(\mathrm{mol} / \mathrm{l}),\left[\mathrm{S}_{\mathrm{A}}\right]$ and $\left[\mathrm{S}_{\mathrm{B}}\right]$ : Concentration of $\mathrm{A}$ and $\mathrm{B}(\mathrm{mol} / \mathrm{l}), \mathrm{E}$ and $\mathrm{F}$ : sigmoidal parameters 
Therefore the chosen flux equation was a simple multiplication of the amino acid concentration in the arterial blood by the blood flow and the fractional uptake ratio, according to the "push" hypothesis, which considers the mammary glands as a passive organ. This flux equation was consequently a straight utilization of the measurements made on the animals. However, the "pull» hypothesis, which assumes an active influence of the mammary gland metabolism on the flux (Knight et al, 1994) was also taken into account by a variable fractional uptake ratio, depending on the milk production level. Therefore this parameterization was a mix between a mechanistic approach and available measurements.

In conclusion, one rule should be followed: the background of a parameter in a biological model should be deeply questioned before it is included (Emmans, 1995).

\section{Numerical integration of the system}

The differential equations which are obtained by translation of the model in mathematical terms can hardly ever be solved analytically because of their nonlinear structure. Therefore they have to be solved numerically by considering these differential equations as finite difference equations (Steiner et al, 1990). Numerical integration of differential equations can be run easily with problem free algorithms. Therefore, obtaining results is no longer dependent on the integration method if the software is correctly chosen (van Milgen et al, 1996).

\section{Parameterization}

\section{Initial compartment sizes}

Each compartment has an initial size. They are the values at the beginning of the simulation, which is the first day of lactation.

According to the definition of the protein compartment, it includes all protein in the organism except those in the blood and the reproductive organs. The problem is to find one reliable method to determine this compartment size and which has been used successfully on lactating cows. Several methods are discussed by Gibb et al (1992) who concluded that the most accurate method was slaughter and subsequent chemical analysis. Two limits were underlined: the uniqueness of the measurement on the same animal and the difficulty to create a time-series during the lactation since a heavy serial slaughter was needed. Nevertheless, a size of the protein compartment could be determined as a percentage of live weight or empty body weight.

The second size to be determined is the reproductive organs one. This includes the reproductive organs after parturition and the colostrum proteins. The total sizes of the reproductive organs are known from embryological studies. The proportion of protein in the reproductive organs can also be determined from the observations of Belyea et al (1978) concerning the composition of the growth in a late-lactation cow. The size of the colostrum pool was deduced from the composition and from the quantity of the colostrum produced. Therefore, compartment initial condition is not precisely known.

The blood amino acid compartment is a metabolic one which is physically located in the blood volume. It is easy to determine the amino acid concentration in this compartment. However, plasma or blood concentration has to be chosen since their amino acid dynamics are different (Backwell, 1994). Another point is to define the compartment volume. Surprisingly, there are no relevant published values of blood volume in the lactating cow. This missing value underlines the lack of quantitative knowledge on areas which were thought to be explored. Since the amino acids are also acting through their concentrations, the failure to predict the compartment volume and its hypothetical variations can weaken the relevance of the model.

The definition of these initial values shows some aspects of the modelling process through the necessity to question the relevance of the available data and through their uncertainty which have to be kept in mind to evaluate the model (Emmans, 1995).

\section{Equation parameter values}

\section{Stoichiometric parameters}

Some fluxes are directly a part of metabolic pathways. Therefore, these parameters are given by the biochemical pathway. However, there are commonly several metabolic routes to obtain one product from one substrate. Urea 
synthesis in the liver has a large set of possibilities concerning the origins of the two ammonia groups and the partition mechanisms are not known (Lobley et al, 1995). Stoichiometric parameters can be obtained from statistical fitting from a specific range of data sets and they should only be used in this range of values (Murphy et al, 1982). This example underlines the relative relevance of a stoichiometric parameter which can be assumed to be constant and is in fact variable.

Parameters obtained from the literature or from experiments

The different biological phenomena represented by the fluxes have been observed at least qualitatively and from time to time quantitatively. However, the first step in determining the parameters is to collect published values and to attempt to define quantitative laws characterizing the parameter variations according to related factors. Obtaining accurate relationships is not often possible due to the heterogeneity of available observations. Moreover, the use of a parameter depends on a sound understanding of the related factors.

As an example, amino acid uptake by the mammary glands, expressed as a fractional rate, is variable depending on the level of milk production and on the amino acid considered with a first statistical approach (Figure 3a, Lescoat and Sauvant, 1994). However, these relationships were obtained by pooling data measured either on blood and on plasma and the blood values were systematically lower both for milk production and for the fractional uptake rate compared with the plasma ones. By discarding the blood values, the previous models were no longer valid (Figure $3 b$, Lescoat et al, 1996). Therefore, in the model the fractional amino acid uptake rate should be assumed constant. Nevertheless, the amino acid fractional uptake was chosen variable to obtain relevant simulations (see section "General behaviour").

A second example is the prediction of protein fractional synthesis rate. Several factors such as the tissue considered, the utilised amino acid, the precursor pool and the nutritional level are influencing its values (Lescoat et al, 1995). Moreover, amino acid recycling in the cell is not taken into account and degraded proteins are recycled for de novo synthesis (Smith and Sun, 1995). There- fore the choice of a value is troublesome. Additionally, the limit between the protein compartment and the blood compartment is not clear. Many data are available to predict the protein turnover rate. However, they are not suited to build quantitative laws. Therefore to determine the anabolic and catabolic fluxes, a data set (Belyea et al, 1978) was fitted with a function, which includes the theoretical hormonal effects, defining these fluxes. This function took into account the nutritional and hormonal effects even though it is not straight. This data set had several drawbacks. First of all, the results from low and high producing cows are averaged. Secondly cows in their first lactation and in the following ones were not discriminated. These averages smooth the curves and consequently hide factors. The authors underlined individual variations but it is not possible to study them since the original raw data were not available. Another weak aspect of this data set is that the independent variable, viz dry matter intake, is not in the tables. Consequently, even though a quite reasonable fitting is made, it is highly dependent on a network of hypotheses. A validation of this theoretical function is needed with new data set like this of Gibb et al (1992).

A major problem in calculating the parameters in compartmental models is overparameterization. An example is the protein anabolic flux; there are at least three parameters. Moreover, the parameters for the catabolic flux have to be determined simultaneously due to the available data set. Therefore, six values are fitted from a data set of eighteen observations. To calculate these parameters, non linear procedures were used: the PROC NLIN of SAS (SAS, 1987) or the SimuSolv package (Steiner et al, 1990). The algorithms estimating the parameters need initial values for these values. Consequently, the estimates obtained could be only valid on a restricted range of utilization and there is the danger that they are linked with a local minimum of the function determining the parameters (Jacquez and Perry, 1990). One solution would be to decrease the number of parameters according to the fact that those parameters are highly correlated and that it is mathematically easier to study a model with a few number of parameters. However, biologists prefer to keep every parameter due to their physiological relevance. Nevertheless, when a limited number of observations are available 


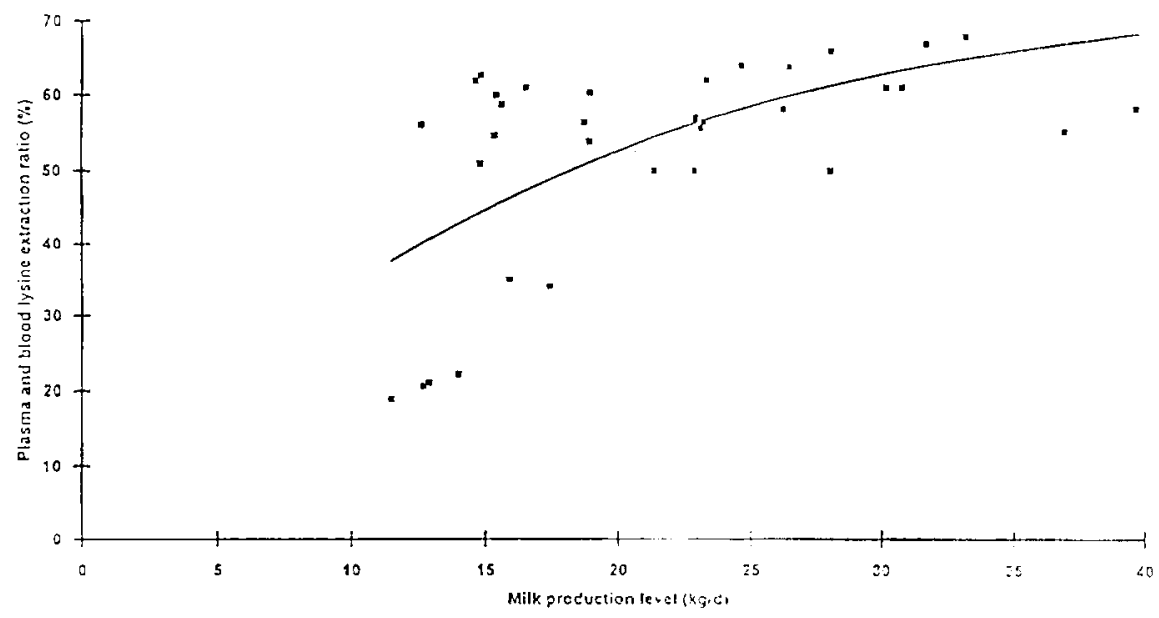

Figure 3a. Lysine extraction ratio in the mammary gland with data on whole blood and plasma.

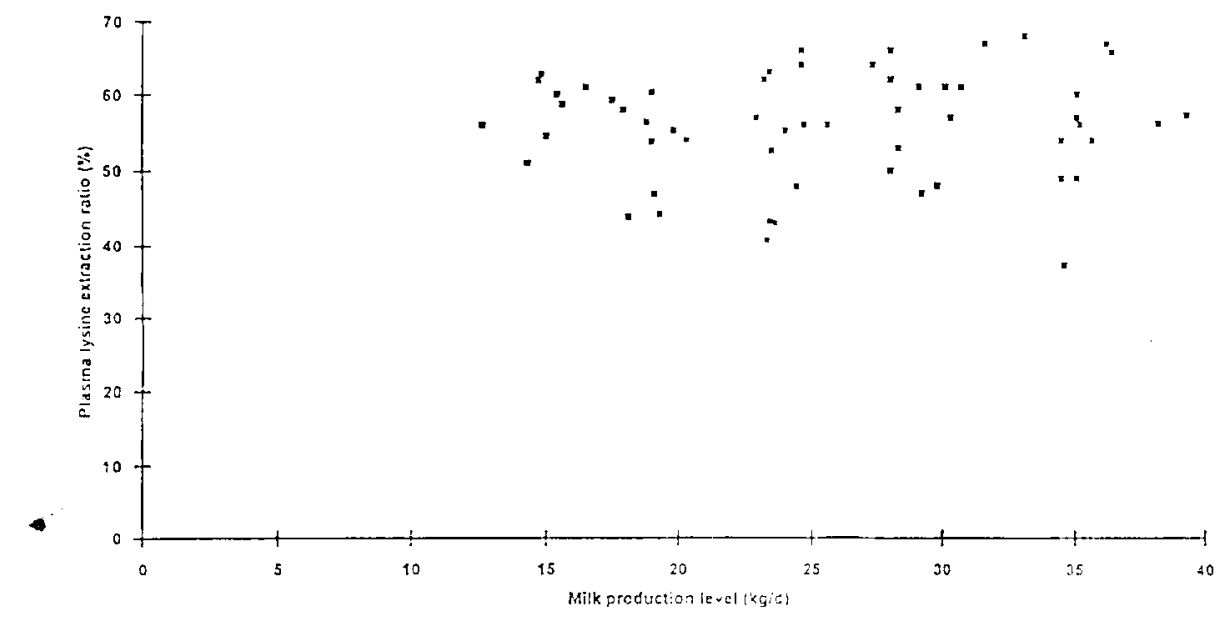

Figure $\mathbf{3 b}$. Lysine extraction ratio in the mammary gland with data on plasma. 
for the fitting step, each value has an high influence on the parameter estimates. Therefore, the qualitative study of the validity of each observation should be reminded. Quantitative tests can be done to underline the influencing values. Iterative estimations, such as the Jackknife method seem an interesting way to avoid biased estimations of the parameters and to decrease the uncertainty if there are enough homogeneous data (Tomassone et al, 1992).

The production of new data is an alternative way to cope with the overparameterization problem. An iterative procedure can be followed. A preliminary structure of the model is proposed and the parameters are evaluated with the available data. From this study, relationships between parameters appeared. Therefore an experimental design could be proposed to increase the knowledge on key-parameters. From the results of this first design, the preliminary structure can be adapted and the sequence experimentation-validation repeated. This approach is also done to evaluate optimal design to avoid an useless sampling procedure. Therefore both the parameters and experimental design are improved (DiStefano, 1981). However, this option implies several experiments and this is time and resources consuming. An experimental approach is also required if several assumptions about the model structure cannot be discriminated between. Since the time and money costs to create new values are high, an alternative way is to generate data from existing ones. For examples, from kinetic curves, values could be estimated by interpolation. This has been done for glucose and insulin kinetics in goats (Tomassone, unpublished results).

\section{Guessed parameters}

Some of the parameters, due to the lack of available data or to their theoretical meaning, could not be estimated: they are not identifiable. For example, the deamination fractional ratio of amino acids in individual tissues of the lactating cow has not been yet estimated. This parameter represents the first step in chemical pathways which could lead to oxidation. The definition of identifiability can be summarized as a parameter (and more generally a model) can be said "identifiable" if it can be estimated mathematically with the available data set and if the solution obtained is unique (Jacquez and Perry, 1990). If too few data are available to identify a parameter, a data simulation process could be done (see section "Parameters obtained from the literature of from experiments") which can discriminate between several solutions on a mathematical standpoint. However, data simulation could strengthen bias on a biological standpoint. Another way to estimate the parameters is to use the Jackknife method. Therefore, smaller data sets are needed for a similar knowledge of the parameters (Jones and Carberry, 1994). However, in this method the initial data set should be carefully examined. If the values are not homogeneous, since the number of observations is limited, the new estimates could be worse that the initial ones (Murphy et al, 1982).

An alternative solution in front of a lack of data on the studied animal is to use either data from other species or in vitro data. However, for the former it can be argued that metabolism can be different in each species like for glucose in monogastrics and ruminants (Danfær et al, 1995). For the latter, as the metabolic control of the pathways are systemic properties (Kacser and Burns, 1979), the use of in vitro results should be done cautiously as in vivo regulation can act differently in in vitro system. The use of in vitro data is done by Baldwin et al (1987a). They stated that the utilization of these data was possible since they found that their model was not highly sensitive to large variations in the parameter estimates. However, they admitted that in vitro experiments can lead to surprising parameter values.

Therefore, the last solution to determine some parameters is to guess their values. These parameters are called "pertinent". Several guidelines can help to define them. Firstly, boundaries can be set i.e. the parameters can only vary in a limited range according to their biological meaning. Secondly, the parameters must be consistent with their connected fluxes. For example, the partition coefficient of the deaminated amino acids between gluconeogenesis and ketogenesis should lead to a biologically relevant value for gluconeogenesis, keeping in mind that amino acid only represent a limited contribution to this flux. Thirdly, these parameters should allow the model to behave soundly (see section "General behaviour»). 
Therefore an iterative process can help to refine the pertinent parameter values. However, the main failure in the guessed values is that their range of validity and their variations are unknown even though a sensitivity analysis can be done on those parameters (see section "Sensitivity analyses to the parameter estimates»).

To conclude, the definition of the parameters is a critical step in a model building. Moreover, it underlines its limits by showing lack of knowledge on some areas of the model. Nevertheless, it opens the way for model analysis and validation.

\section{Internal validation}

\section{General behaviour}

The model is in its early stage and it could simulate only protein mobilization and storage, the milk protein synthesis, the amino acid fate between gluconeogenesis and ketogenesis during the lactation period.

This step of the modelling process consists on analysing if the model is able to represent a lactation of a cow. Therefore, the model is set up for a cow of $600 \mathrm{~kg}$ at the beginning of lactation with a peak of milk production at $30 \mathrm{~kg}$. These inputs calibrate the model and the goal is to compare the outputs with the accepted knowledge on this cow. Hanigan and Baldwin (1994) define these behavioural analyses as a test to know "whether or not equation forms in the model are adequate to simulate observed response patterns." This can be called "black-box" testing due to the fact that nothing between the inputs and the outputs is studied. However, a well oriented "black-box" testing can underline some model weaknesses (Boston et al, 1995).

This step helps to refine parameter estimates. For example, in our model, the milk protein content curve is not satisfactory (Figure 4) and therefore the connected parameters have to be questioned. At least three components can explain this result. Firstly, the blood flow estimation can underestimate the real values. This seems unlikely according to our quantitative review of the literature (Lescoat et al, 1996). Secondly, the amino acid uptake fractional rate, which was constant, influences the quantity taken by the mammary glands. However, as expected from the "pull" hypothesis (Knight et al, 1994), the assumption of a constant fractional uptake has to be rejected and an alternative function has to be

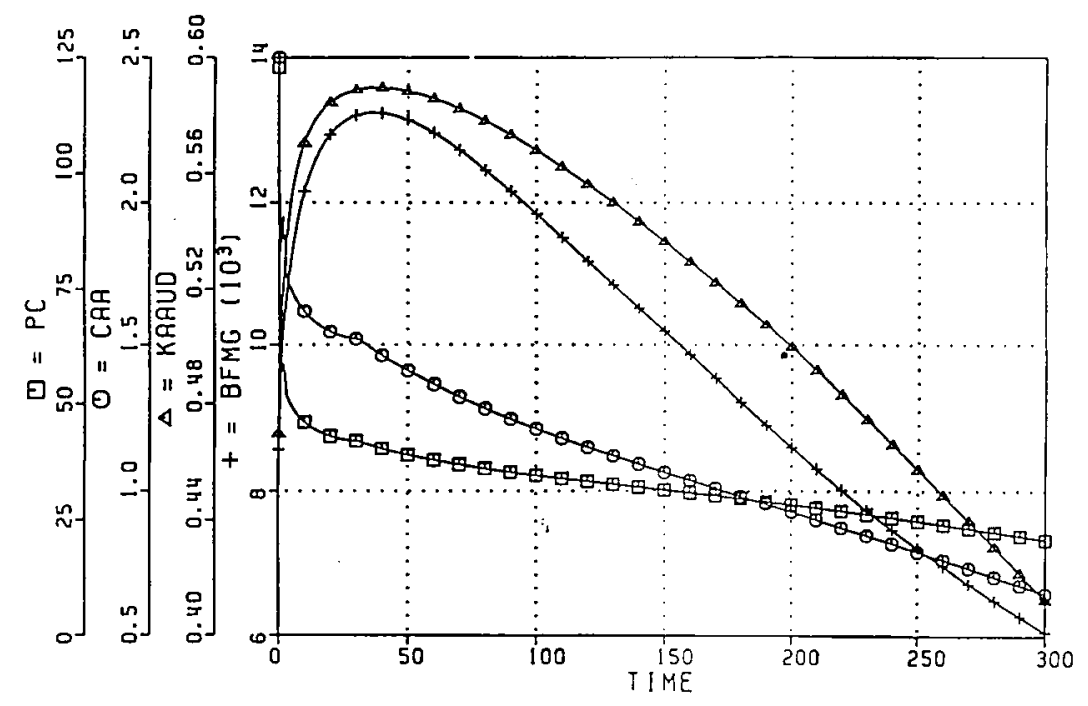

Figure 4. Milk protein content ( $P C, g / k g)$, amino acid blood concentration (CAA, mmol/l), fractional amino acid uptake ratio in the mammary gland (KAAUD), blood flow in the mammary gland (BFMG, $1 /$ d) during the lactation. 
found. Thirdly, blood amino acid concentration influences the available quantity for milk protein synthesis. In our simulation, it decreased to low values. The blood amino acid compartment is a metabolite compartment connected with all the different aspects of the model and consequently its variations are complicated to explain. Therefore, a "white box" analyses (Boston et al, 1995) should be done on the subsystem defining the blood amino acid compartment (see section "Sensitivity analyses to the parameter estimates").

This failure of the model on the amino acid flux in the mammary gland raises the question of the necessity to increase the mechanisticity of this part of the subsystem if more knowledge is available. This could be supported by two recent works of Cant and McBride (1995) and France et al (1995). The former team built a mathematical model to link nutrient uptake with the blood flow in the mammary gland and they concluded qualitatively in a possible local action influencing the quantity taken up. The latter group fitted a model to describe isotopic leucine partition in the mammary glands. Alternative model constructions are therefore possible.

To conclude, this general behaviour step helps to highlight weaknesses in the model structure and by an iterative process to correct them.

\section{Sensitivity analyses to the initial compartment sizes}

The initial conditions to be tested are the size of the compartments. Therefore, three values have to be evaluated: the sizes of the protein compartment, of the reproductive organs and of the blood amino acid compartment. The aim is to test if variations in these compartment sizes will induce perturbations in the model behaviour. The reproductive organs does not need to be studied since they are only important at the start and at the end of lactation and they are much smaller than the protein compartment.

For the protein compartment, a theoretical study of the sensitivity of the model to variations in this compartment would be to change its size by a range of values. This range is limited because the uncertainty cannot exceed $15 \%$, since protein content in the animal is genetically determined. Therefore, the multiplying value to study the stability of the model to the initial conditions range from 85 to $115 \%$ of the reference value. This is in agreement with Baldwin et al (1987a) who stated that parameters do not need to be studied out of their variation space.

Indicators are needed to quantify the perturbations induced by the size variation. The two fluxes from and to the protein compartment are linked with the blood amino acid compartment. Therefore, the blood amino acid concentration seems to be a relevant indicator of the perturbation. Moreover, since these fluxes are high, the influence of the protein compartment size can be studied directly on some outputs of the blood amino acid compartment, as the milk protein synthesis, the amino acid utilization for gluconeogenesis and for ketogenesis.

The blood amino acid initial size should be studied. However, its daily turnover flux is several hundred times its size, large variations of its initial value would therefore have no influence on the model behaviour. Instead of the blood amino acid compartment size, the blood diffusion volume seems to be the initial condition to be studied since it determines the blood amino acid concentration. Moreover the uncertainty of this value is high (see section "Initial compartment sizes»). Consequently, a sensitivity analysis can be done by multiplying the blood volume by a factor ranging from 75 to $125 \%$ of the reference value. This effect can be quantify on several outputs of the model like milk protein synthesis and gluconeogenesis.

To conclude, a step by step approach is used for sensitivity analyses. Firstly, the compartments to be studied have to be identified. Secondly, a range of variations have to be decided according to the uncertainty of the reference value. Thirdly, the outputs of the model able to represent the perturbation induced by the change in the initial conditions have to be isolated.

\section{Sensitivity analyses to the parameter estimates (Table II)}

The model must react to parameter variation to mimic a biological system. A main point of the sensitivity analyses is to determine if these variations are in a reasonable range. Moreover, since most of the parameters have a given uncertainty, sensitivity analyses must 
Table II. Sensitivity analyses approaches.

$\begin{array}{cc}\begin{array}{c}\text { Group of } \\ \text { parameters }\end{array} & \begin{array}{c}\text { Knowledge about the } \\ \text { coefficients } \\ \text { Regulating } \\ \text { subsystem } \\ \text { Protein }\end{array} \\ \begin{array}{c}\text { Theoretical } \\ \text { compartement }\end{array} & \begin{array}{c}\text { Fitted to the regulating } \\ \text { subsystem and to } \\ \text { published values }\end{array}\end{array}$

Blood amino acid Measured or estimated compartment

\author{
Variations of the \\ coefficients \\ Unknown, guess of \\ pertinent limits \\ Biological \\ background
}
Availiable standard deviations

\author{
Related outputs \\ Proetin and lipid \\ compartments \\ Protein \\ compartment and \\ fluxes, amino acid \\ fate \\ Output from the \\ compartment
}

be done to quantitate their influence on the model.

The first step is to determine the parameters to test. Those from reproductive organ compartment can be neglected because of the limited influence of this compartment on the model. Three sets of parameters have to be evaluated: each parameter of the protein compartment or the blood amino acid one or those of the regulating subsystem.

It has to be decided if the three sets of parameters have to be analyzed simultaneously. To our knowledge, modelling softwares do not offer the possibility to test simultaneously the sensitivity of a model for several parameters (Steiner et al, 1990). Since the appropriate statistical tools are not available for non-linear equations, difficulties in testing hypotheses defining the interactions between parameters are appearing. However, as Pettigrew et al (1992) stated «The parameters are interrelated, so that a change in one would logically be accompanied by changes in others, further complicating and extending an exhaustive test". A solution is to divide the system into subsections by assuming independence between subsystems. The regulating subsystem can be analysed on one side whereas the blood amino acid compartment and the protein one have to be studied together. Moreover, the fluxes of the blood amino acid compartment can be influenced by the protein compartment fluxes but the reverse does not occur. Thus, three sets of parameter sensitivity analyses have to be conducted: the regulating subsystem, the blood amino acid compartment parameters alone and the blood amino acid and protein compartments together.
A range of values has to be decided on for each parameter. This choice is based on the knowledge of this parameter (Baldwin et al, 1987a). To study the perturbations induced by these changes, outputs have to be chosen. For example, since the regulating subsytem drives the protein compartment, output will be fluxes of this compartment. However, since they also influence energy metabolism, milk fat and protein synthesis can be relevant indicators (Figure 5). The goal is to find the closest flux or variable influenced by the tested parameter. An additional problem with this model is that it covers the whole lactation period. Therefore, the period of the output should be any day during the simulation. This was not the case in rumen steady-state models (Lescoat and Sauvant, 1995) where the outputs were aggregated sums at the end of the simulation period. To solve this time-dependent sensitivity, a two-step proposal can be tested for the lactating cow model. Firstly, each output can be added from the beginning to the end of lactation. For example, protein compartment anabolism would be represented by the sum of the protein compartment inputs during the whole lactation. From this first approach, the tested parameters can be qualified to influence the whole lactation simulation process. Secondly, the sensitivity of the model can be tested on a day-by-day approach. This possibility is well documented in the SimuSolv package (Steiner et al, 1990) where they defined this sensitivity as local opposed to a global sensitivity (Figure 5).

For parameters that can be studied alone, the usual approach of changing the value of this parameter and observing the models behaviour seems sufficient. For example, 
Neal et al (1992) gave most of the tested parameters a range of values from -50 to +50 $\%$ from the reference one with three different inputs. However, when interrelations are suspected, attempts can be made to study their changes simultaneously. In a rumen model (Lescoat and Sauvant, 1995), five keyparameters were studied together in a complete factorial design. The statistical tests were not valid since the model tested were non linear. However, if the Fisher Test values were high, then the significance of the effect could be qualitatively accepted. From this analysis, interactions were highlighted. An extension to this approach would be to build a variancecovariance matrix to link the different parameters and to create an optimal design to study their influences. For the lactating cow model, the approach is limited to the one proposed by Lescoat and Sauvant (1995).

To conclude, the internal validation steps are in fact several steps to test the relevance of the model structure (Neal et al, 1992), its stability to initial conditions and parameter changes.

\section{External validation}

\section{Compartment by compartment analyses}

Each compartment can tested as a "blackbox" by being challenged against non previously utilized data. However these data are very difficult to find because most of the relevant values have been used to calibrate the model. For example, Freetly et al (1993) had to validate their liver model with in vitro data because of the use of all available in vivo data for the model building steps. An alternative solution has been proposed by Jones and Carberry (1994) if all the available data were used: Jackknife and crossvalidation. Those methods estimate the parameters and validate the model from an initial data set. This is allowed by the use of resampling techniques which consist of taking subgroups of the whole data set in an iterative manner. This approach was also proposed by Aumont et al (1993 and unpublished results) who validated com-partmental models of ruminal digestion by the use of the Jackknife method using simulated data sets.

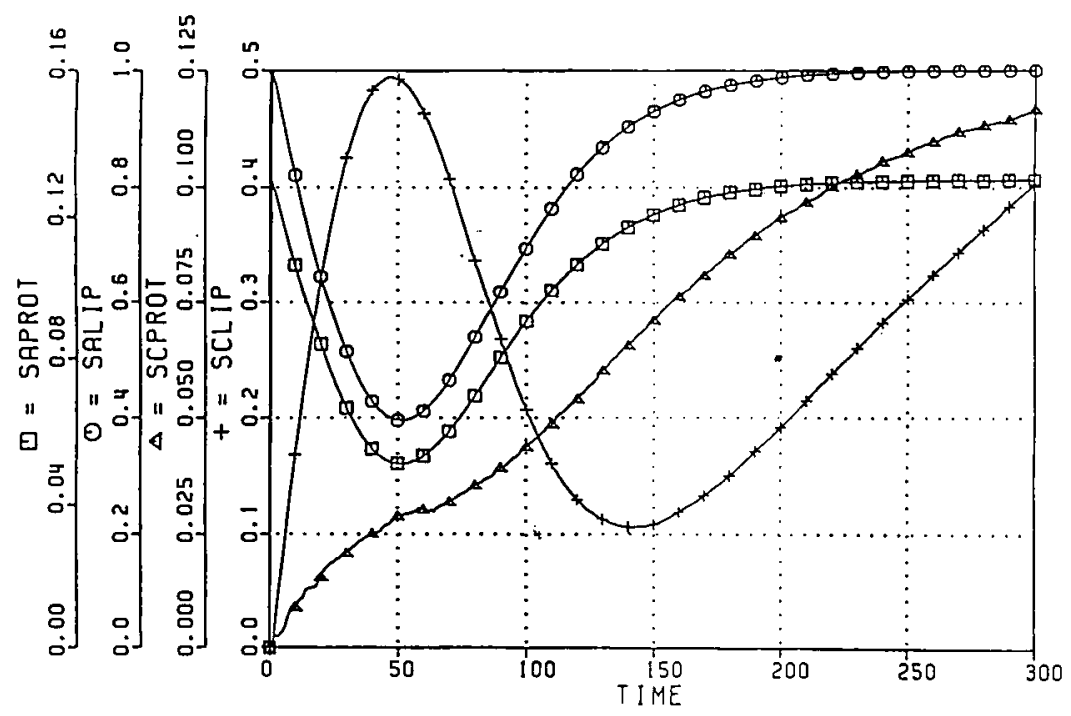

Figure 5. Sensitivity analyses of the anabolic (SAPROT, SALIP) and the catabolic (SCPROT, SCLIP) fluxes of protein and lipid to the ratio Rma between the anabolic (HA) and the catabolic (HM) hormonal effects ( $H A=R m a / H M)$. 
In the protein section of the lactating cow model, three compartments can be validated. The reproductive organ compartment requires the time-course of the decrease in the reproductive organ size during the first month of lactation and their increase in the last gestation months. These observations should be compared graphically with simulated curves of reproductive organ size evolution. Because of the early stage of the model, there is no need for a quantitative comparison on this small compartment.

For the protein compartment, a new set of data was available in the literature during the model development. A large slaughter experiment was conducted on 54 cows during their first 29 weeks of lactation. Moreover, three different levels of concentrate were given to the cows (Gibb et al, 1992). Therefore, three observed curves of the protein compartment size can be compared with the simulated ones. However, no validation of the anabolism or the catabolism fluxes can be directly done due to the fact that the data are an addition of the two processes. Nevertheless, a validation of the protein compartment can be made since curves are available for different diets. The best would be to have individual values of this experiment to highlight the model limits. However, the validation conclusions with these values support that new protein compartments should be added, like the portal drained viscera, and these values can be used to parameterize these changes.

The blood amino acid compartment is an aggregation of most phenomena regarding amino acid metabolism. Therefore, attempts can be done to validate each flux. First of all, the inputs from the diet can be evaluated. Theoretically, this validation is easy since these inputs were directly calculated from the French PDI system equations slightly modified (INRA, 1988). However these amino acids are transported through the intestinal wall before they reach the portal blood and the extent of the portal drained viscera withdrawal of amino acids has to be taken into account. Therefore, two sources of data can be used for the validation; either the PDI equations or the portal blood amino acid appearance. In the former case, there is no need for validation since these equations have been successfully used for years. The relevance of comparing portal appearance and intestinal lumen disappearance is questioned (MacRae et al, 1995) and therefore the input in the blood amino acid compartment is not set up yet.

A second flux to validate is the deamination step. However, few results are available (Danfær et al, 1995) and they have been used to parameterize these fluxes. An alternative way would be to study urea production, as product of deamination. However, Lobley et al (1995) highlighted that the origin of the ammonia groups in urea is not clear and therefore in our lactating cow model assumptions should be added to take into account urea production.

The mammary gland amino acid uptake is the last output to be studied for this compartment. However, this validation will consist on comparing the uptake of amino acids and their output in milk protein. Therefore the problem of the accuracy of this approach will be questionned since the extent of the amino acid metabolism in the mammary gland is not precisely known (France et al, 1995).

The state variable, i.e. the size of the compartment, can be studied through the amino acid concentration in whole blood which is obtained directly by dividing directly the compartment size by the blood volume. The simulated concentration can be followed during the whole lactation and compared with results of so-called "amino acid concentration". However, the blood amino acid compartment includes the amino acids and small peptides both attached to the red blood cells and in plasma according to our definition. Moreover, recent results show that the method used for amino acid or peptide analysis has a large effect on the results obtained (Bernard and Rémond, 1995). Therefore, this simulated amino acid concentration can be qualitatively compared with the measured ones bearing in mind the measurements uncertainties.

This compartment by compartment external validation can be seen as an iterative process inducing the following stages of model development: at each validation, if the obtained results are not satisfactory at a compartment level, the model parameter or structure are transformed.

\section{Outputs of the model}

The final step in each loop of a modelling process is the external validation of the model. 
New data that have not been used in building the model are needed. This statement is systematically quoted in published models to improve a priori confidence in the validation (Pettigrew et al, 1992; Freetly et al, 1993). New observations are available because they are too aggregated to support the internal model parameterization. For example, many field trials which have investigated milk production and composition and dry matter intake are available to validate the lactating cow model. However, selected data have to be checked for their relevance. For example, in the rumen model of Baldwin et al (1987b), the model was challenged against data from animals fed a diet with a high level of barley and therefore the fit was not accurate because the model was not calibrated for such extreme diets.

Additionally, the model outputs have to be defined in agreement with the model target and the available data. Since the whole-model is treated like a "black-box", the available outputs are those out of the model limits. For example, in the lactating cow model, these outputs are milk protein production and body weight.

However, the main problem is to adapt these observations to the model. Depending on both the precision of the inputs needed to run the model and the available information in the observations, a network of assumptions have to be done to calculate these inputs. Therefore, the comparison of the simulated and the obtained results can be weakened by a large uncertainty linked with the definition of the input values (Sørensen, 1990).

When the model can be challenged against new data, an additional issue is to evaluate the "goodness of fit" (Sørensen, 1990). In the lactating cow model, a graphical comparison between observed and simulated curves during the whole lactation seems a first way. Sorensen (1990) proposed at least two qualitative tests on time series like in the lactating cow model. The first is based on the comparison between the simulated and observed time series for their numbers of turning points (local minimum and maximum), the timing of turning points and their amplitude (distance from local minimum to next local maximum). The second qualitative test is the Turring one; the observed and simulated time series are presented to external experts and they are asked to distinguish between the two series. The latter approach seems of high relevance due to the fact that a team has built a model with a set of hypothesis and as it is a long process, the model builders have integrated these assumptions and are less objective than external expert.

After these qualitative tests, quantitative objective tests are needed. In models where the outputs are obtained at the end of the simulation period, for examples the live weight change during lactation in the sow (Pettigrew et al, 1992), a comparison between the simulated and the measured outputs gives a quantitative validation of the model. The determination and decomposition of the mean square prediction error (Bibby and Toutenburg, 1977 ) is a reliable tool to evaluate a model. It indicates if there is an overall bias, a bias due to an initial parameterization or a misconception in the equations of the model. This tool can be used for a local evaluation of the lactating cow model. For example, if several measured observations are available on day 30 of the simulation, then the validity for this day can be checked by this tool. However, for the whole lactation period, the graphical approach seems sufficient even though it is a subjective one due to the early stage in the development of this model.

The best solution is to challenge the model in an actual trial. For example, in a pig growth model, Moughan et al (1987) designed an experiment with 100 growing pigs and compared real and simulated results. The main bottleneck is to build an experiment which will increase knowledge on the model's features. For the lactating cow model, a production trial during the whole lactation period with different diets and if possible non invasive estimation of the fat and protein pools would be valuable. However, these experiments for the purpose of modelling are almost never done due to the associated time and monetary costs. Practical alternatives are to challenge the model on each new data set made available and therefore either increase the confidence in the model or decrease it and to take part in the planning of experiments by adding some measurements which could help for the model validation.

For the lactating cow model several approaches are available for the validation 
steps. Their use will depend on the future development of the model.

\section{Conclusion}

From this paper, it appears that a model is a sum of assumptions implying limits in its utilization. A model pinpoint areas where there is a lack of knowledge by stressing a high number of questions. Therefore, it can be an important tool to design experiments and to aggregate the available knowledge. However, it could not prove that results are wrong a point that is in conflict with the proposal of Hanigan and Baldwin (1994).

The process of building and validating a model is a heuristic one with a high number of iterations to improve the structure and the systemic properties of the model. Therefore the modelling process generates a systemic behaviour of the modeller, implying feedbacks between each step and inside each of them. There is no strict rule to determine if a step is achieved. Subjective model builder's statements are "the simulated results are reasonable" or "the model behaves soundly". They only highlight the fact that the authors consider that they have reached an intermediary step from which they can present their up-to-date version of the model. Cornerstone examples are given by the work of the team of $\operatorname{Pr} R L$ Baldwin at the University of California (Davis). They have proposed new versions of their cow model regularly since 1970.

Finally, it has to be kept in mind that modelling approaches need the use of mathematical tools and physical or chemical concepts. Therefore, since each of these tools is not complicated for the mathematician or the chemestrian but is not always understandable for nutritionists who wants to build a cow model, the modelling approach will gain if it is done in an interdisciplinary group (Greco,1986).

Building of relevant whole-cow metabolism models is a long-term process. The question of whether these models can be of some help to build new feedstuff evaluation systems is open to debate. This paper can be a contribution to this exchange of ideas thanks to the discussion of the complexity of the processes involved in modelling animal metabolism.

\section{Acknowledgements}

The authors acknowledge Vince M Gabert, National Institute of Animal Science (Denmark) and University of Alberta (Edmonton, Canada) for correcting the English. We thank $D^{R} P$ Williams, director of research and $D^{R} \mathrm{JC}$ Robert, director of ruminant research and development at Rhône Poulenc Animal Nutrition (Antony, France) for their financial and scientific support.

\section{Literature cited}

Aumont G, Cerneau I, Xande A (1993) Effects of the repeatability of nitrogen degradability. Consequences for the estimation of protein values of tropical forages in French unit system. In: "XIII Réunion ALPA", 22-31 july 1993, Santiago, Chili

Backwell FRC (1994) Peptide utilization by tissues: current status and applications of stable isotope procedures. Proc Nutr Soc 53, 457-464

Baldwin RL, France J, Gill M (1987a) Metabolism of the lactating cow I. Animal elements of a mechanistic model. J Dairy Res 54, 77-105

Baldwin RL, Thornley JHM, Beever DE (1987b) Metabolism of the lactating cow II. Digestive elements of a mechanistic model. J Dairy Res 54, 107-131

Battaglia FC (1992) New concepts in fetal and placental amino acid metabolism. J Anim Sci 70, 3258-3263

Belyea RL, Frost GR, Martz FA, Clark JL, Forkner LG (1978) Body composition of dairy cattle by Potassium-40 liquid scintillation detection. J Dairy Sci 61, 206-211

Bernard L, Rémond D (1995) Effect of two methods of deproteneization on the estimation of peptide bound amino acids in whole blood or plasma. In: VII Symposium on Protein Metabolism and Nutrition, 24-27 May 1995, Estacao Zootecnica Nacional, Vale de Santarem, Portugal, 139

Bibby J, Toutenburg H (1977) Prediction and improved estimation in linear models. John Wiley and Sons, London, England

Boston RC, Ferguson JD, Kohn RA, Black JL (1995) Model testing and evaluation. In: Proceedings of IVth International Workshop on Modelling Nutrient Utilisation in Farm Animals (A Danfær and P 
Lescoat, eds) National Institute of Animal Science, Foulum, Denmark, 25-34

Cant JP, MacBride BW (1995) A mathematical analysis of the relationship between blood flow and uptake of nutrients in the mammary glands of a lactating cow. J Dairy Res 62, 405-422

Danfær A (1990) A dynamic model of nutrient digestion and metabolism in lactating dairy cows. Thesis, Report 671, National Institute of Animal Science, Foulum, Denmark, $511 p$

Danfær A, Tetens V, Agergaard N (1995) Review and an experimental study on the physiological and quantitative aspects of gluconeogenesis in lactating ruminants. Comp Biochem Physiol 111B, 201-210

DiStefano J (1981) Optimized blood sampling protocols and sequential design of kinetic experiments. Am J Physio/ 240, R259-R265

Emmans GC (1995) Models and the iterative process of developing and testing hypotheses. In: Proceedings of IVth International Workshop on Modelling Nutrient Utilisation in Farm Animals (A Danfær and $P$ Lescoat, eds) National Institute of Animal Science, Foulum, Denmark, 19-24

France J, Bequette BJ, Lobley GE, Metcalf JA, WrayCahen D, Dhanoa MS, Backwell FRC, Hanigan MD, MacRae JC, Beever DE (1995) An isotope dilution model for partitioning uptake by the bovine mammary gland. $J$ theor Biol $172,369-377$

Forbes JM, France J (1993) Introduction. In: Quantitative aspects of ruminant digestion and metabolism (JM Forbes and J France, eds) CAB International, Wallingford, England, 1-10

Freetly HC, Knapp JR, Calvert CC, Baldwin RL (1993) Development of a mechanistic model of liver metabolism in the lactating cow. Agric Sys 41, 157-195

Gibb MJ, Ivings WE, Dhanoa MS, Sutton JD (1992) Changes in the body components of autumncalving Holstein-Friesian cows over the first 29 weeks of lactation. Anim Prod 55, 339-360

Greco WR (1986) The role of simulation in biomathematical modeling. Bull math Biol 48, 241251

Hanigan MD, Baldwin $R L$ (1994) A mechanistic model of mammary gland metabolism in the lactating Cow. Agric Sys 45, 369-419

INRA (1988) Alimentation des bovins, ovins, caprins ( $R$ Jarrige, ed) Institut National de la Recherche Agronomique, France, $471 \mathrm{p}$

Jacquez JA, Perry T (1990) Parameter estimation : local identifiability of parameters. Am J Physiol 21, E727-E736

Jones PN, Carberry PS (1994) A technique to develop and validate simulation models. Agric Sys 46, 427-442
Kacser H, Burns JA (1979) Molecular democracy: Who shares the controls?. Biochem Soc Trans 7 , 1149-1160

Knight CH, France J, Beever DE (1994) Nutrient metabolism and utilization in the mammary gland. Livest Prod Sci 39, 129-137

Le Moigne JL (1990) La modélisation des systèmes complexes. Afcet systèmes, Dunod, Paris, France, $178 \mathrm{p}$

Lescoat P, Sauvant D (1994) Amino acid uptake predictions in the mammary gland of the lactating cows. Proc Soc Nutr Physio/ 3, 238

Lescoat P, Sauvant D (1995) Development of a mechanistic model for rumen digestion validated using the duodenal flux of amino acids. Reprod Nutr Dev 35, 45-70

Lescoat P, Danfær A, Sauvant D, Ponter AA, DuvauxPonter C (1995) A statistical analyses of protein turnover rate in ruminants. In: VII Symposium on Protein Metabolism and Nutrition, 24-27 May 1995, Estacao Zootecnica Nacional, Vale de Santarem, Portugal, 120

Lescoat P, Sauvant D, Danfær A (1996) Quantitative aspects of blood and amino acid flows in cattle. Reprod Nutr Dev (in press)

Lobley GE, Connell A, Lomax MA, Brown DS, Milne E, Calder AG, Farningham DAH (1995) Hepatic detoxification of ammonia in the ovine liver: possible consequences for amino acid metabolism. Br J Nutr 73, 667-685

MacRae JC, Backwell FRC, Bequette BJ, Lobley GE (1995) Protein metabolism in specific tissues. In: VII Symposium on Protein Metabolism and Nutrition, 24-27 May 1995, Estacao Zootecnica Nacional, Vale de Santarem, Portugal, 98

Moughan PJ, Smith WC, Pearson G (1987) Description and validation of a model simulating growth in the pig (20-90 kg liveweight). New Zealand J Agric Res 30, 481-489

Murphy MR, Baldwin RL, Koong LJ (1982) Estimation of stoichiometric parameters for rumen fermentation of roughage and concentrate diets. $J$ Anim Sci 55, 411-421

Neal HD St C, Dijkstra J, Gill M (1992) Simulation of nutrient digestion, absorption and outflow in the rumen: model evaluation. J Nutr 122, 2257-2272

O'Connor J, Sniffen C, Fox D, Chalupa W (1993) A net carbohydrate and protein system for evaluating cattle diets. IV Predicting amino acid adequacy. J Anim Sci 55, 411-421

Pettigrew J, Gill M, France J, Close WH (1992) Evaluation of a mathematical model of lactating sow metabolism. J Anim Sci 70, 3762-3773

Rulquin H, Pisulewski PM, Verité R, Guinard J (1993) Milk production and composition as a function of postruminal lysine and methionine supply: a 
nutrient-response approach. Livest Prod Sci 37 , 69-90

SAS/STATIM guide for personal computer: Statistics, Version 6 Edition (1987) SAS Inst Inc, Cary, NC, USA, $1028 p$

Sauvant D (1994) Modelling homeostatic and homeorhetic regulations in lactating animals. Livest Prod Sci 39, 105-113

Smith CB, Sun $Y$ (1995) Influence of valine flooding on channeling of valine into tissue pools and on protein synthesis. Am J Physiol 268, E735-E744

Steiner EC, Rey TD, McCroskey PS (1990) SimuSolv modeling and simulation software. The Dow Chemical Company, Midland, Michigan

Sørensen JT (1990) Validation of livestock herd simulation models: a review. Livest Prod Sci 26 , 79-90

Tagari H, Bergman EN (1978) Intestinal disappearance and portal blood appearance of amino acids in sheep. J Nutr 108, 790-803

Tamminga $S$, van Straalen WM, Subnel APJ, Meijer RGM, Steg A, Wever CJG, Blok MC (1994) The Dutch protein evaluation system: the DVE/OEBsystem. Livest Prod Sci 40, 139-155

Tomassone R, Audrain S, Lesquoy de Turckheim E, Millier $C$ (1992) La régression linéaire, nouveaux regards sur une ancienne méthode statistique. INRA Masson, Paris, France, $188 \mathrm{p}$

van Milgen J, Boston R, Kohn R, Ferguson J (1996) Comparison of available sottware for dynamic modeling. Ann Zootech 45, suppl, 257-273 